\title{
The Origin of the Lithium Rich Giants
}

\author{
Corinne Charbonnel \\ Laboratoire d'Astrophysique de l'Observatoire Midi-Pyrénées, Toulouse, \\ France
}

Suchitra Balachandran

University of Maryland, USA

\begin{abstract}
We use Hipparcos parallaxes to determine the evolutionary status and the nature of the so-called Li-rich giants. These informations clearly support the hypothesis of internal nuclear lithium enrichment by an extra-mixing process at two distinct evolutionary phases.
\end{abstract}

\section{The so-called Li-rich giants}

About $1 \%$ of the G-K giants show unexpected strong lithium lines, some of these so-called Li-rich RGB even presenting abundances higher than the present interstellar medium value. Different propositions have been made to explain the Li-rich giant phenomenon. Some are related to external processes, like the contamination of the external layers of the giant by the debris of nova ejecta or by the engulfing of a planet. Other explanations refer to internal processes, like the preservation of the initial lithium content or fresh lithium production (see also De la Reza in this volume).

\section{Clues on the nature of the Li-rich giants}

When available, we use the Hipparcos data to determine the mass and evolutionary status of the Li-rich giants of the literature by comparing their position on the HRD with theoretical evolutionary tracks (Fig.1; see Charbonnel \& Balachandran 2000). Among the twenty objects of the sample for which a precise determination of the location was possible, 8 appear to be Li-rich simply because they have not completed the standard first dredge-up dilution, and must thus be reclassified as Li-normal; these stars are the hexagons on the figure.

The remaining stars are confined in two special places in the HRD. First, the four stars with the highest $\mathrm{Li}$ abundance (black circles) are located at the place referred to as the bump in the luminosity function (shaded area). At this evolutionary point the outwardly moving hydrogen burning shell passes through the mean molecular weight discontinuity created by the first dredge-up. This permits access to a reservoir of ${ }^{3} \mathrm{He}$ rich material by an extra-mixing process which is actually known to significantly decrease the carbon isotopic ratio in lowmass RGB stars (e.g., Charbonnel et al. 1998, Charbonnel et al. in this volume). 


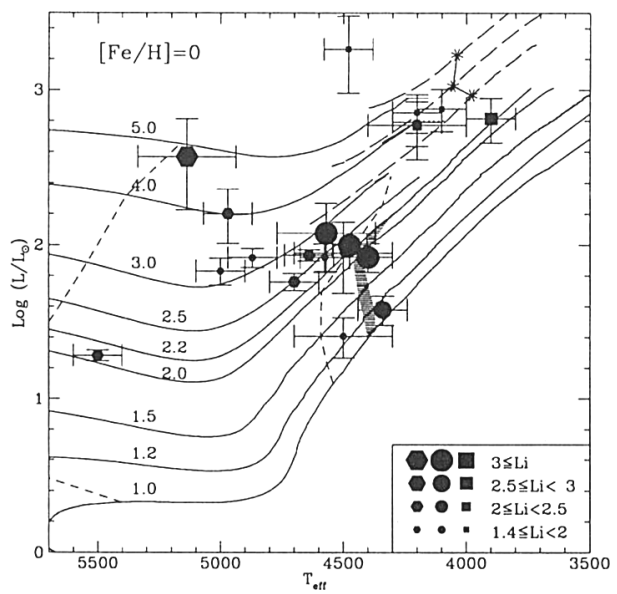

Figure 1. HR diagram for the Li-rich giants. The evolutionary tracks for $[\mathrm{Fe} / \mathrm{H}]=0$ are labelled by their mass. The short dashed lines and the asterisks delimit the first and second dredge-up respectively. The shaded region surrounds the location of the bump

We thus bring to the fore and clearly identify an episode of Li production at the RGB bump. Since no Li-rich RGB of low mass has been identified above the bump, we can conclude that the freshly synthetized $\mathrm{Li}$ is destroyed very quickly, as soon as the extra-mixing extends deep enough to change the surface carbon isotopic ratio.

Finally, five Li-rich stars are intermediate mass objects (black squares) which have ignited central He burning in a non-degenerate core before reaching the RGB bump. They are at present located on the early-AGB (dashed lines), close to the completion of the second dredge-up (asterisks). We thus identify an episode of $\mathrm{Li}$ production at this phase where the convective envelope is deepening but has not yet build a new molecular weight barrier between its base and the hydrogen burning shell; we expect the barrier to stop the extra-mixing so that this Li-rich episode is limited both in time and luminosity. We do not endorse any specific mechanism for the extra-mixing as none is evident from the observations.

Since both Li-rich episodes are very short, these stars should not contribute significantly to the Li enrichment of the ISM. We refer to Charbonnel \& Balachandran (2000) for a more complete discussion.

\section{References}

Charbonnel C., Balachandran S., 2000, A\&A in press, astro-ph/0005280

Charbonnel C., Brown J.A., Wallerstein G., 1998, A\&A 332, 204

Charbonnel C., Deliyannis C.P., Pinsonneault M., 2000, this volume 